\title{
Pathway Complexity in Fuel-Driven DNA Nanostructures with Autonomous Reconfiguration of Multiple Dynamic Steady States
}

\author{
Jie Deng ${ }^{\mathrm{a}-\mathrm{d}}$, and Andreas Walther ${ }^{\mathrm{a}-\mathrm{d} *}$ \\ ${ }^{a} A^{3}$ BMS Lab, Institute for Macromolecular Chemistry, University of Freiburg, Stefan-Meier-Straße 31, 79104 Freiburg, \\ Germany \\ ${ }^{\mathrm{b}}$ DFG Cluster of Excellence "Living, Adaptive and Energy-Autonomous Materials Systems" (livMatS), 79110 Freiburg, \\ Germany \\ ${ }^{\mathrm{c}}$ Freiburg Materials Research Center, University of Freiburg, Stefan-Meier-Straße 21, 79104 Freiburg, Germany \\ ${ }^{\mathrm{d}}$ Freiburg Center for Interactive Materials and Bioinspired Technologies, University of Freiburg, Georges-Köhler-Allee 105, \\ 79110 Freiburg, Germany
}

\begin{abstract}
We introduce pathway complexity on a multicomponent systems level in chemically fueled transient DNA polymerization system. The systems are based on a monomeric species pool that is fueled by ATP and orchestrated by an enzymatic reaction network (ERN) of ATP-powered ligation and concurrent cleavage. Such systems display autonomous evolution over multiple structural dynamic steady states from monomers to dimers, oligomer of dimers to ultimately randomized polymer structure before being ultimately degraded back to monomers once the fuel is consumed. The enabling key principle is to design monomer species having kinetically selected molecular recognition with respect to the structure-forming step (ATP-powered ligation) by encoding different sticky-end overhangs into the ligation area. However, all formed structures are equally degraded, and the orthogonal molecular recognition of the different starting species are harmonized during the constantly occurring restriction process, leading in consequence to a reconfiguration of the driven dynamic nanostructures on a higher hierarchical level. This non-equilibrium systems chemistry approach to pathway complexity provides new conceptual insights in fuel-driven automatons and autonomous materials design.
\end{abstract}

Nature relies on non-equilibrium structures and processes to perform work by dissipating chemical energy stored in ATP or GTP. ${ }^{1-}$ ${ }^{4}$ Pathway complexity plays a pivotal role to associate those dynamic structures and thus realize a living system. For instance, intracellular signaling networks involve multiple steps of ATP/GTPpowered phosphorylation and enzyme activation for downstream regulations. ${ }^{5-7}$ Inspired from ubiquitous processes in nature where energy-driven assembling structures offer a variety of intriguing possibilities, ${ }^{8}$ synthetic systems with fuel-driven transient properties are developed in the fields of systems chemistry, materials science and synthetic biology. ${ }^{4-12}$ Synthetic systems requiring continuous energy influx have extended the features and functions of present-day functional materials. ${ }^{13-14}$

Although several non-equilibrium systems have been reported so far, as for instance for helical structure switching, ${ }^{15}$ supramolecular polymerization, ${ }^{14,16}$ nanoreactors, ${ }^{13,}{ }^{17}$ transient hydrogels, ${ }^{18-19}$ and photonic materials, ${ }^{20}$ such fuel-driven structures mostly show single trajectories of their non-equilibrium states. On the contrary, in classical supramolecular chemistry, pathway complexity is regularly exploited to transiently pass from one metastable structure into the next one. ${ }^{21-22}$ Therein, however, all systems follow downhill equilibration. A relevant example showing multiple states in an autonomous fashion is the case of supramolecular helicity switching coupled to hydrolysis of ATP to ADP, AMP, and to adenosine. ${ }^{15}$ The consecutive, but indirect switching in this dissipative environment system is programmed via consecutive degradation of an environmental signal. In directly chemically driven systems,
Boekhoven and coworkers recently showed that small fuel amounts allowed the formation of reversibly assembling transient clusters, while large fuel amounts lead to a trapping of aggregates in a nontransient fashion. ${ }^{23}$ Critically, pathway-controlled uphill fueldriven systems involving multiple building blocks with an autonomous structural reconfiguration of multiple dynamic transient states have not been shown by any previous report. Such a concept would however be very valuable to promote a further integration of systems chemistry concepts with reaction networks and dissipative and transient structure formation.

The difficulty in implementing such a concept in synthetic system is related to the need for highest levels of programmability and ability for deterministic autonomous reconfiguration leading to multiple transient dynamic steady-state (DySS) structures, which is a profound challenge to achieve in supramolecular chemistry field. ${ }^{24-26}$ Contrary to classical supramolecular building blocks, DNA shows prominent programmability concerning the hybridization interactions, which provides great opportunities to program reaction networks and pathways for self-assemblies in a systems chemistry approach. ${ }^{27-29}$

Our system builds on our recently reported ATP-fueled DySS polymerization of DNA nanostructures using an uphill-driven dynamic covalent DNA bond orchestrated by an enzymatic reaction
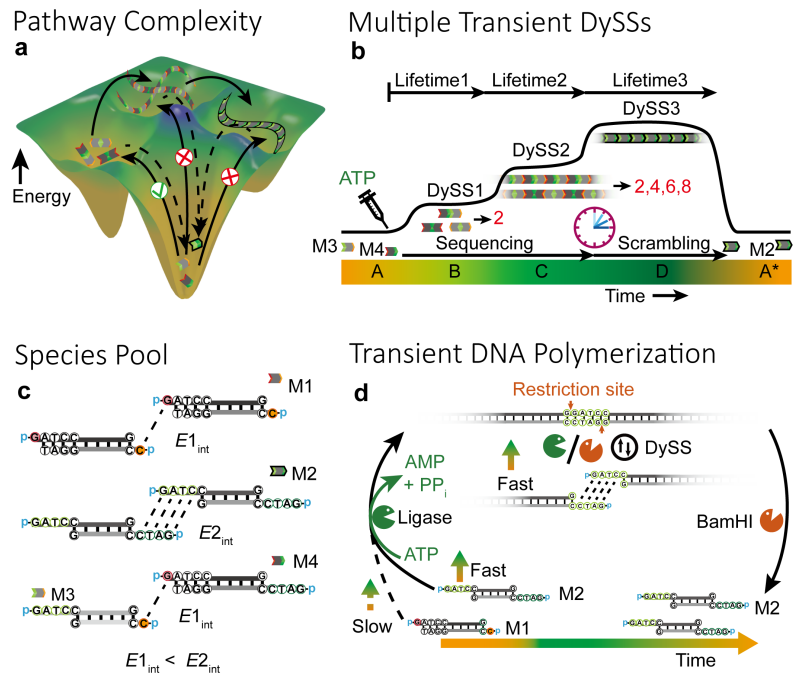

Transient DNA Polymerization

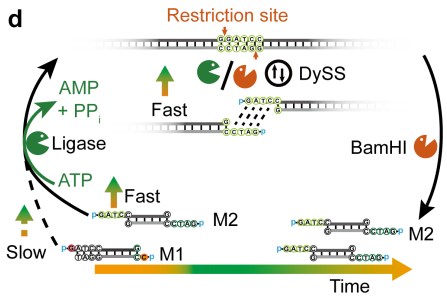

Figure 1. Pathway complexity in ATP-powered transient multi-state systems using a species pool of reconfigurable elements. (a) Schematic illustration of pathway complexity in ATP-driven DNA polymerization. (b) ATP-driven autonomous multiple transient DySSs. (c) Building blocks with varied length of sticky ends. (d) Schematic illustration of transient polymerization of $1 \mathrm{nt}$ and $4 \mathrm{nt}$ sticky-end dsDNA tiles. Note the reconfiguration of the $1 \mathrm{nt}$ ends into $4 \mathrm{nt}$ ends. 
network (ERN) of concurrent ligation by T4 DNA ligase and BamHI-controlled cleavage of dsDNA building blocks bearing sticky-end overhangs (Figure 1d). ${ }^{30}$ Importantly, BamHI constantly generates a standard $4 \mathrm{nu}-$ cleotide (nt) self-complementary sticky end, 5'-GATC-3'. In striking contrast, the molecular recognition for the ATP-powered ligation at the restriction zone can in fact be changed in its length, which implies that different complementary recognition pairs from the starting species pool can be kinetically selected. We hypothesized that combinations of tiles with sticky ends of different length can induce different pathways by distinct recognition and binding strength, and then a reconfiguration occurs via uniformization of the sticky ends in the ERN as time progresses (Figure 1d; Figure S1).

Building on this hypothesis, we demonstrate pathway complexity in ATP-fueled transient DNA polymerizations via the combination of species containing $1 \mathrm{nt}$ and $4 \mathrm{nt}$ sticky ends (or combinations of those), aiming to achieve autonomous and transient mulitple DySS structures (Figure 1a,b). Figure 1c shows the species pool for this study. Specifically, the 1 nt sticky ends in M1 show lower stickiness than the $4 \mathrm{nt}$ sticky ends in M2. M1 and M2 cannot be ligated, thus providing orthogonal molecular recognition. Additionally, the kinetics for the ligation of M1 are much slower than that for M2. The tiles with combined $1 \mathrm{nt}$ and 4 nt sticky ends, M3 and M4, serve as the key for pathway complexity in the system due to the kinetically selective sequential ligation of these sticky ends (4 nt ends faster than $1 \mathrm{nt}$ ends). Subsequently, uniformization of the sticky ends occurs in the ERN due to BamHI.

We first demonstrate the basic principle of the concept by programming lag times into the autonomous lifecycles of the DySS DNA polymers by shortening the ligatable sticky ends from previously $^{30}$ used $4 \mathrm{nt}$ to $1 \mathrm{nt}$ (M1), aiming for a lag time before the transient state due to much lower stickiness of $1 \mathrm{nt}$ sticky end and lower efficiency of the ligation (Figure 1d). Agarose gel electrophoresis (AGE) of time-dependent aliquots visualizes the transient nature of the formed DySS DNA polymers regarding their length distributions. As expected, $4 \mathrm{nt}$ sticky-end tiles, M2, display a fast and direct growth phase. The chains rapidly grow to ca. $800 \mathrm{bp}$ within 10 min and reach a DySS plateau at ca. 9000 bp after 15 min (Figure 2c; Figure S2). In striking contrast, for the transient polymerization of M1 there is no significant chain elongation for the first hour (Figure 2a). This system can only enter the growth phase after substantial completion of the M1 conversion phase, during which the $1 \mathrm{nt}$ sticky-end overhangs are increasingly converted to 4 nt sticky-end overhangs (hence become M2) to promote ligation efficiency. This is increasingly achieved after ca. $1 \mathrm{~h}$ of transient polymerization, yet, only after $5 \mathrm{~h}$, there is an obvious gel band shift to higher molecular weights.

Upon reaction progress the mutual feedback of both processes in the ERN leads to a balance in the DySS and to a constant frequency of ligation and restriction. Once the ATP fuel is increasingly consumed, the ligation process seizes and the kinetic balance shifts towards the degradation. After four days, the DySS DNA polymers are degraded and the both systems decompose to the monomer state; M2 in both cases. A quantification of the AGE allows to calculate the average mass-weighted chain length in base pairs $\left(\overline{b p}_{w}\right)$ from the mass-weighted distribution. The DySS DNA polymers from $\mathrm{M} 1$ show ca. $1000 \mathrm{bp}$ lower $\overline{b p}_{w}$ than the DNA polymers from
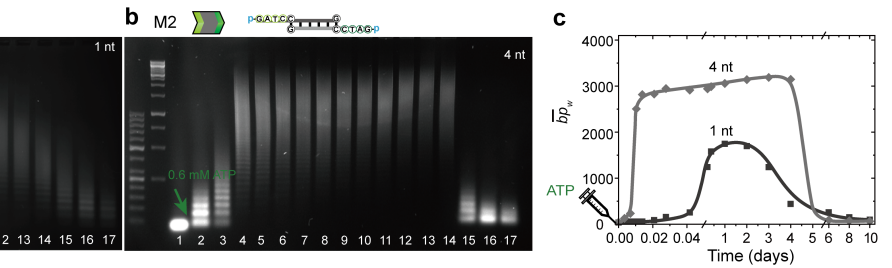

th controlled lag time in ATP-driven, dynamic covalent DNA assembly. (a,b)
t. $\%, 90 \mathrm{~V}, 2 \mathrm{~h})$ and (c) development of $\overline{b p}_{w}$ for the transient polymerization of M1 and M2 by fueling with $0.6 \mathrm{mM}$ ATP. Line are guides to the eye. Conditions: $25^{\circ} \mathrm{C}, 1 \times$ Promega Buffer E, 0.05 mM M1 or M2, 0.46 WU(Weiss Unit) $/ \mu \mathrm{L}$ T4 DNA ligase, 10 units/ $\mu \mathrm{L}$ BamHI, and $0.6 \mathrm{mM}$ ATP. Lane assignment: 1: $0 \mathrm{~min}, 2: 5 \mathrm{~min}, 3: 10 \mathrm{~min}, 4: 15 \mathrm{~min}, 5: 20 \mathrm{~min}, 6: 30 \mathrm{~min}, 7: 40 \mathrm{~min}, 8: 1 \mathrm{~h}, 9: 5 \mathrm{~h}, 10: 9 \mathrm{~h}, 11: 1 \mathrm{~d}, 12: 2 \mathrm{~d}$,

M2 (Figure 2b,d), which is attributed to a continuous evolution of M1 to M2 at the DySS and thus slowed down ligation. Overall, the implementation of a conversion phase allows to implement a lag time control for the transient DNA polymerization by shortening the ligatable overhangs from $4 \mathrm{nt}$ to $1 \mathrm{nt}$. It is worth noting that a 2 nt or 3 nt overhang cannot induce an obvious lag time for the transient DNA polymerization (Figure S3).

Next we investigated higher levels of pathway complexity of the fuel-driven self-assembly. From above, it is evident that 4 nt stickyend double-stranded DNA (dsDNA) tiles can be ligated much faster than $1 \mathrm{nt}$ sticky-end dsDNA tiles. Hence, we designed two asymmetrical dsDNA tiles bearing $1 \mathrm{nt}$ and $4 \mathrm{nt}$ overhangs on their terminal parts, M3 and M4 (Figure 3). The $1 \mathrm{nt}$ overhangs on M3 and M4 are complementary to each other (5'-G/C-3') while the $4 \mathrm{nt}$ overhangs on M3 and M4 are the same and self-complementary (5'-GATC/GATC-3'). We assumed that temporally selective ligation of the overhangs on these asymmetrical dsDNA tiles will happen during fueling of the ERN with ATP. Due to the lag time for 1 nt overhangs ligation, first, a dimer state forms by the ligation of the 4 nt overhangs, leading to a DySS1. Then, the dimers act as new monomers for the temporally secondary polymerization of the DNA tiles connected by 1 nt overhangs ligation, resulting in a second DySS of oligomers of dimers (DySS2), being representative of a different fuel-driven uphill localized minimum in the energy landscape. Subsequently, M3 and M4 evolve in the ERN to standard tiles with 4 nt overhang (M2) after one complete cycle of dynamic ligation and cleavage. Thus, the sequenced DNA oligomers from DySS1 and DySS2 are scrambled in this long lasting and

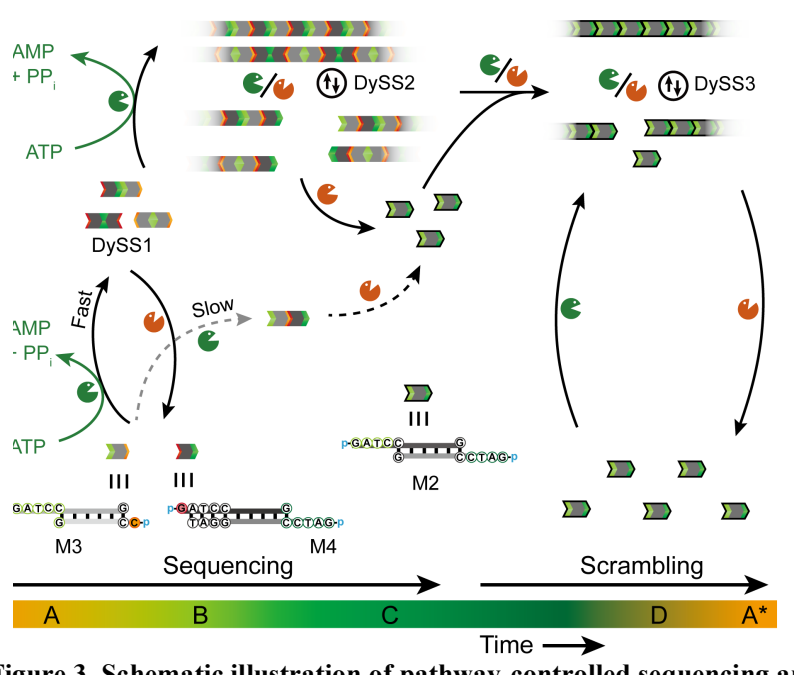

Figure 3. Schematic illustration of pathway-controlled sequencing and scrambling for autonomous multiple transient DySSs Polymerization of dsDNA tiles bearing 1 and 4 nt overhangs leads to sequencing of the system to dimers via ligation of $4 \mathrm{nt}$ overhangs and afterwards oligomers of dimers, which are scrambled after tiles evolution in the ERN. 
conversion phase, enabling the formation of DNA polymers with randomized numbers of initial monomers in DySS3. All DNA polymers degrade to $4 \mathrm{nt}$ overhang dsDNA tiles (M2) after the ATP is consumed. Hence, this concept demonstrates pathway-controlled sequencing and scrambling that leads to the formation of multiple transient DySSs.

Indeed, AGE visualizes the transient multi-state nature of the systems fueled by $0.3,0.6$ and $0.9 \mathrm{mM}$ ATP being driven consecutively into three different DySSs (Figure 4a-c). For instance, by fueling with $0.3 \mathrm{mM}$ ATP, the ligation brings the monomers into a dimer state (DySS1), showing a lifetime of ca. $30 \mathrm{~min}$ as defined by the time when longer oligomers appear. Then the system is in DySS2 by chain extension of the dimers from DySS1, leading to the formation of oligomers of dimers, and the system stays in DySS2 for ca. $30 \mathrm{~min}$. Afterwards, the system leaves the conversion phase and autonomously enters the DySS3, polymer phase with scrambled oligomers. Gray scale profiles extracted from AGE at $0.6 \mathrm{mM}$ ATP show more direct evidence for the sequential sequencing and scrambling effects by transient polymerization of M3 and M4 (Figure 4d). Below 30 min, the gel band only shifts to the position for dimer. Then at $1 \mathrm{~h}$, the gray scale profile shows sharp peaks for all the oligomers with even numbers of monomers. After $5 \mathrm{~h}$, new peaks for the oligomers with odd number of monomers appear between the peaks for oligomers with even number of monomers and elongation into polymers takes place. The system fueled with 0.3 and $0.6 \mathrm{mM}$ ATP show a plateau of $\overline{b p}_{w}$ at ca. $1700 \mathrm{bp}$. Increasing the ATP concentration to $0.9 \mathrm{mM}$ further promotes the $\overline{b p}_{w}$ by ca. $400 \mathrm{bp}$ in a sustained DySS polymerization due to larger availability of the ATP concentration (Figure 4e). The lifetimes for DySS1 and DySS2 do not significantly depend on the fuel level, because there is a significant excess of ATP in the beginning, yet the lifetime for DySS3 is almost doubled when increasing the ATP level from $0.3 \mathrm{mM}$ to $0.6 \mathrm{mM}$. Further increasing the ATP concentration to $0.9 \mathrm{mM}$ only slightly shortens the lifetime for DySS1 by $5 \mathrm{~min}$, but the lifetime for the whole process is further increased to ca. 5 days (Figure $4 \mathrm{f}$ ). The reason for the insensitivity of the lifetimes of DySS1 and DySS2 to the ATP concentration is because the sequencing process is programmed by the lag time of $1 \mathrm{nt}$ overhangs ligation and evolution of M3 and M4 to M2 in the ERN, which mainly correlates to the cleavage kinetic in the ERN. Thus, ATP concentration shows very limited effect on the lifetimes for DySS1 and DySS2.

Afterwards, we investigated whether it would be possible to disrupt this sequencing effect by adding $\alpha, \omega$ telechelic 4 nt-overhang dsDNA tiles (M2) to the above transient polymerization system with asymmetrical tiles (M3 and M4) to further influence the transient structural evolution. We performed the experiments the same as above (Figure $4 \mathrm{~b} ; 0.6 \mathrm{mM}$ ATP), except using different ratios of M3:M4:M2 (1:1:0, 5:5:2, and 1:1:1). Compared to the system without M2 (1:1:0, Figure 4b), the introduction of $16.7 \mathrm{~mol} \% \mathrm{M} 2$ (5:5:2) completely eliminates DySS2, and the lifetime of DySS1 is decreased by $10 \mathrm{~min}$ (Figure 5a). Although the introduced M2 can promote the ligation, M3 and M4 in the system can act as terminators for the ligation of M2. Since the amount of M2 is comparably low, homopolymerization of M2 is not pronounced by blocking with M3 and M4, hindering the formation of longer oligomers. After 20 min, the increasing conversion of 1 nt overhangs to 4 nt overhangs leads to elongated oligomers. However, the system does not enter a distinct DySS2, but directly goes to DySS3, because M2 tiles are available to produce odd numbers. A further increase of M2 to $33.3 \mathrm{~mol} \%$ significantly speeds up the polymerization process (Figure 5b) and the lifetime of DySS1 is further decreased by
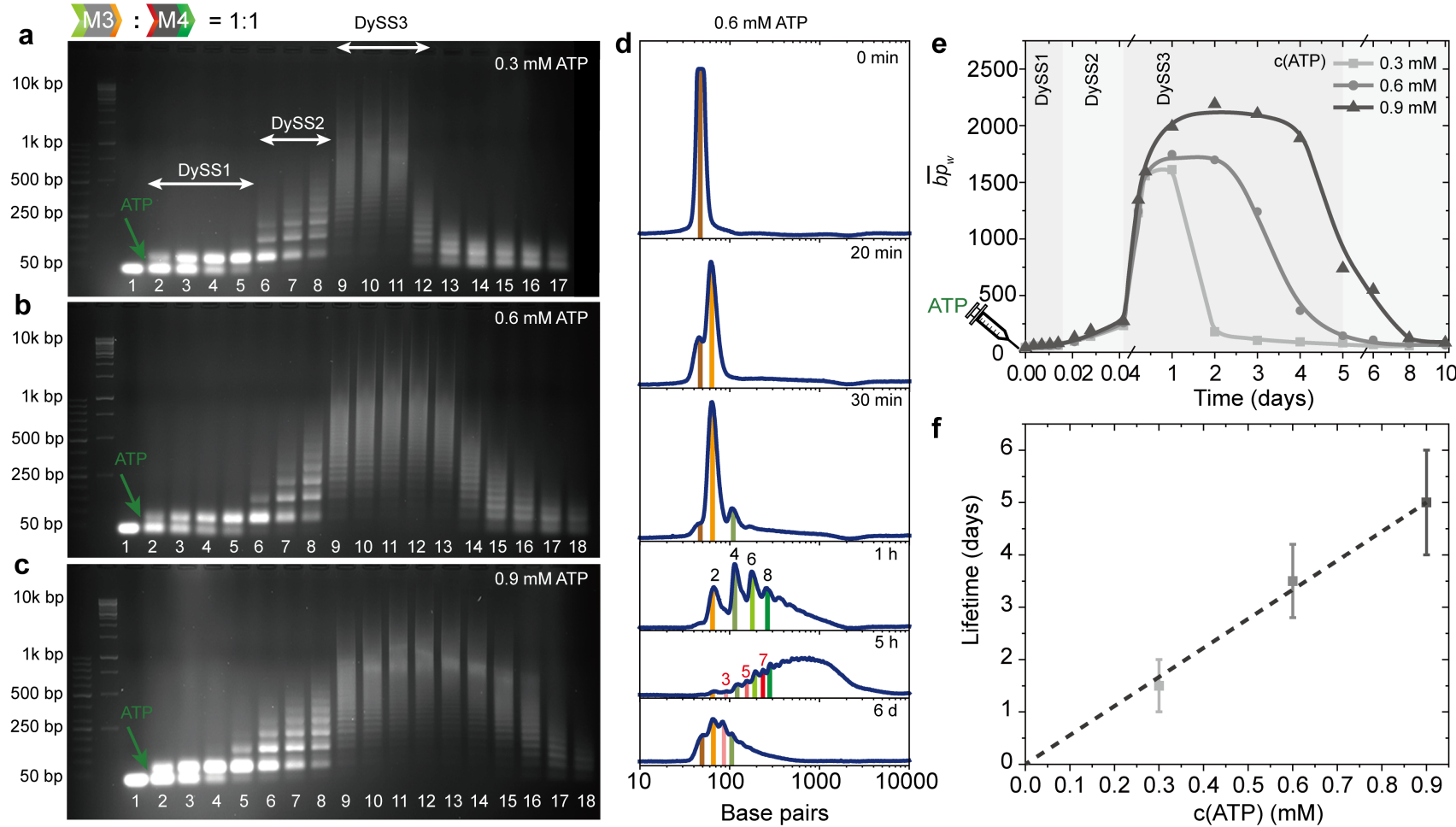

Figure 4. ATP-powered autonomous multiple transient DySS structures. (a-c) Time-dependent AGE ( 2 wt. \%, $90 \mathrm{~V}, 2 \mathrm{~h})$ for the transient DySS polymerizations of M3 and M4 by fueling with (a) 0.3, (b) 0.6, and (c) $0.9 \mathrm{mM} \mathrm{ATP.} \mathrm{(d)} \mathrm{Gray} \mathrm{scale} \mathrm{profiles} \mathrm{from} \mathrm{AGE} \mathrm{for} \mathrm{the} \mathrm{system} \mathrm{in} \mathrm{(b).} \mathrm{(e)} \mathrm{Development} \mathrm{of} \mathrm{the}$ $\overline{b p}_{w}$ with time for the systems (a-c). Lines are guides to the eye. (f) The lifetimes are controlled by the ATP concentration. Error bars are standard deviations of duplicate measurements. Conditions: $25^{\circ} \mathrm{C}, 1 \times$ Promega Buffer E, $0.025 \mathrm{mM} \mathrm{M}, 0.025 \mathrm{mM} \mathrm{M} 4,0.46 \mathrm{WU} / \mu \mathrm{L}$ T4 DNA ligase, 10 units/ $\mu \mathrm{L}$ BamHI, and varied concentration of ATP. Lane assignment: 1: $0 \mathrm{~min}, 2: 5 \mathrm{~min}, 3: 10 \mathrm{~min}, 4: 15 \mathrm{~min}, 5: 20 \mathrm{~min}, 6: 30 \mathrm{~min}, 7: 40 \mathrm{~min}, 8: 1 \mathrm{~h}, 9: 5 \mathrm{~h}, 10: 9 \mathrm{~h}, 11: 1 \mathrm{~d}, 12: 2 \mathrm{~d}$, $13: 3 \mathrm{~d}, 14: 4 \mathrm{~d}, 15: 5 \mathrm{~d}, 16: 6 \mathrm{~d}, 17: 8 \mathrm{~d}, 18: 10 \mathrm{~d}$ 

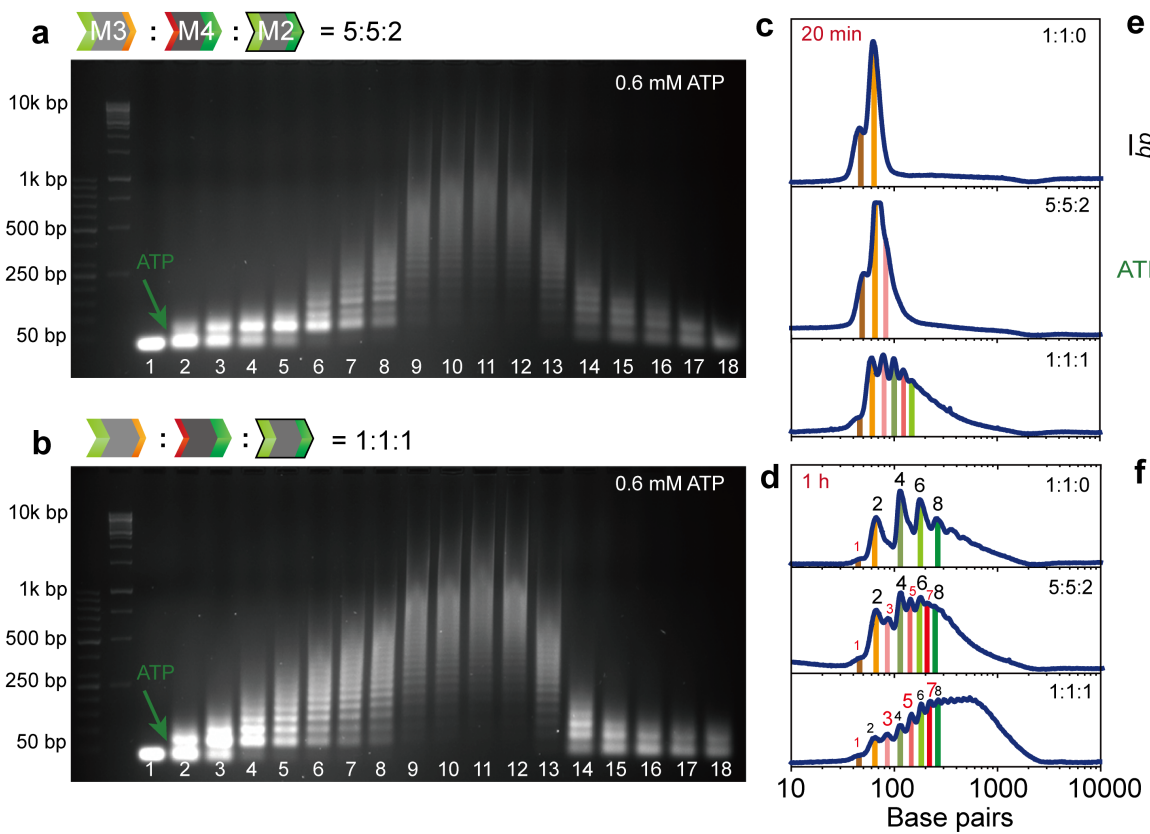

Figure 5. Disruption of programmable transient multi-states. (a-b) Time-dependent AGE ( 2 wt. \%, 90 V, 2 h) for transient polymerizations of M3, M4, and M2 with a ratio of (a) 5:5:2 and (b) 1:1:1:1 by fueling with 0.6 mM ATP. (c-d) Gray scale profiles from AGE at (c) 20 min and (d) 1 h for transient polymerization of M3, M4, and M2 with varied ratios ( $0.6 \mathrm{mM}$ ATP). (e) Development of $\overline{b p}_{w}$ with time for transient DySS polymerizations of M3, M4. and M2 at different ratios (0.6 mM ATP). Lines are guides to the eye. (f) Lifetime control in the disrupted systems for DySS1 and DySS1-3. Error bars are standard deviations of duplicate measurements. Conditions: $25{ }^{\circ} \mathrm{C}, 1 \times$ Promega Buffer $E, 0.05 \mathrm{mM}$ dsDNA with different ratios for M3, M4, and M2, $0.46 \mathrm{WU} / \mu \mathrm{L}$ T4 DNA ligase, 10 units/ $\mu \mathrm{L}$ BamHI, and 0.6 mM ATP. Lane assignment: 1: $0 \mathrm{~min}, 2: 5 \mathrm{~min}, 3: 10 \mathrm{~min}, 4: 15 \mathrm{~min}, 5: 20 \mathrm{~min}, 6: 30 \mathrm{~min}, 7: 40 \mathrm{~min}, 8: 1 \mathrm{~h}, 9: 5$ h, 10:9h, 11:1d, 12:2d, 13:3d, 14:4d, 15:5d, 16:6d, 17:8d, 18:10d.

$20 \mathrm{~min}$. A rather smooth but much slower evolution into the DySS3 occurs as compared to for instance using M2 alone (Figure 2c; Figure S2b). Gray scale profiles extracted from AGE visualize the distribution of the transient DNA polymers. The gray scale plots at 20 min visualize the chain elongation efficiency with increased M2 ratio (Figure 5c). After the dimer state, the polymerized DNA oligomers from M3, M4, and M2 show both even and odd number of monomers. The signals of DNA oligomers with odd number of monomers increase with the increase of $\mathrm{M} 2$ ratio (Figure $5 \mathrm{~d}$ ). The addition of M2 also promotes the $\overline{b p}_{w}$ to higher values in a sustained DySS polymerization as well as fast polymerization after DySS1 (Figure 5e).

A comparison of the lifetimes of the DySS1 shows an inverse linear relationship with the M2 concentration (Figure 5f). However, the whole lifetimes for the autonomous multi-sates transition are mainly correlated to ATP fuel level. The system without M2 shows a bit longer lifetime due to slower overall ligation kinetic by the relatively higher concentration of $1 \mathrm{nt}$ sticky-end overhangs (Figure $5 f)$.

In summary, we introduced an energetically uphill driven autonomous switching of multiple transient DySS structures in a fueldriven system, whereby multiple building blocks from a species pool are reconfigured in a pre-programmable fashion. This pathway complexity on a systems chemistry level has been enabled by programming the recognition and stickiness of the ligation positions in the ATP-powered ligation step (structure forming process), while the BamHI-controlled restriction (structure degradation) homogenizes them. The building blocks with ligatable overhangs of different stickiness show sequential ligation and recognition overhang evolution in the ERN. The set building blocks evolve autonomously into different driven states by reconfiguration of the sticky ends to trigger different assembly scenarios.
This multicomponent systems chemistry approach to chemically fueled pathway complexity contrast earlier work on classical supramolecular pathway complexity (oriented towards equilibrium) and comparably simple one-component chemically fueled systems, that only have limited ability for structural reconfiguration. It paves new avenues for non-equilibrium systems with structurally evolving multiple transient DySS states for higher levels of functions in emerging chemically fueled non-equilibrium systems, automatons and dynamic molecular materials.

\section{ASSOCIATED CONTENT}

\section{Supporting Information}

Full methods and experimental data. This material is available free of charge via the Internet at http://pubs.acs.org.

\section{AUTHOR INFORMATION}

\section{Corresponding Author}

andreas.walther@makro.uni-freiburg.de

\section{Notes}

The authors declare no competing financial interests.

\section{ACKNOWLEDGMENT}

We acknowledge support by the European Research Council starting Grant (TimeProSAMat) Agreement 677960. We thank L. Heinen for establishing the ATP-driven DNA polymerization system and F. Lossada for the help with Blender.

\section{REFERENCES}

1. Van den Heuvel, M. G.; Dekker, C., Motor Proteins at Work for Nanotechnology. Science 2007, 317 (5836), 333-336. 

Reductionist Agenda. Nature 2003, 422 (6933), 741.

3. Mitchison, T.; Kirschner, M., Dynamic Instability of Microtubule Growth. Nature 1984, 312 (5991), 237.

4. Merindol, R.; Walther, A., Materials Learning from Life: Concepts for Active, Adaptive and Autonomous Molecular Systems. Chem. Soc. Rev. 2017, 46 (18), 5588-5619.

5. $\quad$ Taylor, M. J.; Husain, K.; Gartner, Z. J.; Mayor, S.; Vale, R. D., A DNA-based T Cell Receptor Reveals a Role for Receptor Clustering in Ligand Discrimination. Cell 2017, 169 (1), 108-119. e20.

6. Li, L.; Yang, J.; Wang, J.; Kopeček, J. i., Amplification of Cd20 Cross-linking in Rituximab-resistant B-lymphoma Cells Enhances Apoptosis Induction by Drug-free Macromolecular Therapeutics. ACS Nano 2018, 12 (4), 3658-3670.

7. Vaudry, D.; Stork, P.; Lazarovici, P.; Eiden, L., Signaling Pathways for PC12 Cell Differentiation: Making the Right Connections. Science 2002, 296 (5573), 1648-1649.

8. Salazar-Roa, M.; Malumbres, M., Fueling the Cell Division Cycle. Trends Cell Biol. 2017, 27 (1), 69-81.

9. Grzybowski, B. A.; Huck, W. T., The Nanotechnology of Lifeinspired Systems. Nat. Nanotechnol. 2016, 11 (7), 585.

10. van Rossum, S. A.; Tena-Solsona, M.; van Esch, J. H.; Eelkema, R.; Boekhoven, J., Dissipative out-of-equilibrium Assembly of Man-made Supramolecular Materials. Chem. Soc. Rev. 2017, 46 (18), 5519-5535.

11. Sorrenti, A.; Leira-Iglesias, J.; Markvoort, A. J.; de Greef, T. F.; Hermans, T. M., Non-equilibrium Supramolecular Polymerization. Chem. Soc. Rev. 2017, 46 (18), 5476-5490.

12. Mattia, E.; Otto, S., Supramolecular Systems Chemistry. Nat. Nanotechnol. 2015, 10 (2), 111.

13. Maiti, S.; Fortunati, I.; Ferrante, C.; Scrimin, P.; Prins, L. J., Dissipative Self-assembly of Vesicular Nanoreactors. Nat. Chem. 2016, 8 (7), 725

14. Sorrenti, A.; Leira-Iglesias, J.; Sato, A.; Hermans, T. M., Nonequilibrium Steady States in Supramolecular Polymerization. Nat Commun. 2017, 8, 15899.

15. Dhiman, S.; Jain, A.; Kumar, M.; George, S. J., Adenosinephosphate-fueled, Temporally Programmed Supramolecular Polymers with Multiple Transient States. J. Am. Chem. Soc. 2017, 139 (46), 16568-16575.

16. Mishra, A.; Korlepara, D. B.; Kumar, M.; Jain, A.; Jonnalagadda, N.; Bejagam, K. K.; Balasubramanian, S.; George, S. J., Biomimetic Temporal Self-assembly Via Fuel-driven Controlled Supramolecular Polymerization. Nat. Commun. 2018, 9 (1), 1295.

17. Pezzato, C.; Prins, L. J., Transient Signal Generation in a Selfassembled Nanosystem Fueled by Atp. Nat. Commun. 2015, 6, 7790.

18. Heuser, T.; Weyandt, E.; Walther, A., Biocatalytic Feedbackdriven Temporal Programming of Self - regulating Peptide Hydrogels. Angew Chem. Int. Ed. 2015, 54 (45), 13258-13262.
19. Heinen, L; Heuser, T; Steinschulte, A; Walther, A. Antagonistic Enzymes in a Biocatalytic pH Feedback System Program Autonomous DNA Hydrogel Life Cycles. Nano Lett. 2017, 17 (8), 49894995.

20. Heuser, T.; Merindol, R.; Loescher, S.; Klaus, A.; Walther, A., Photonic Devices out of Equilibrium: Transient Memory, Signal Propagation, and Sensing. Adv. Mater. 2017, 29 (17), 1606842.

21. Heinen, L.; Walther, A., Celebrating Soft Matter's 10th Anniversary: Approaches to Program the Time Domain of Self-assemblies. Soft Matter 2015, 11 (40), 7857-7866.

22. Madan, I.; Buh, J.; Baranov, V. V.; Kabanov, V. V.; Mrzel, A.; Mihailovic, D., Nonequilibrium Optical Control of Dynamical States in Superconducting Nanowire Circuits. Sci. $A d v$. 2018, 4 (3), eaao0043.

23. Grötsch, R. K.; Wanzke, C.; Speckbacher, M.; Ang1, A.; Rieger, B.; Boekhoven, J., Pathway Dependence in the Fuel-driven Dissipative Self-assembly of Nanoparticles. J. Am. Chem. Soc. 2019, 141 (25), 9872 9878.

24. Dong, B.; Liu, L.; Hu, C., ATP-driven Temporal Control over Structure Switching of Polymeric Micelles. Biomacromolecules 2018, 19 (9), 3659-3668

25. della Sala, F.; Maiti, S.; Bonanni, A.; Scrimin, P.; Prins, L. J., Fuel-selective Transient Activation of Nanosystems for Signal Generation. Angew Chem. Int. Ed. 2018, 57 (6), 1611-1615.

26. Hao, X.; Chen, L.; Sang, W.; Yan, Q., Periodically Selfpulsating Microcapsule as Programmed Microseparator Via ATP-regulated Energy Dissipation. Adv. Sci. 2018, 5 (3), 1700591.

27. Qian, L.; Winfree, E., Scaling up Digital Circuit Computation with DNA Strand Displacement Cascades. Science 2011, 332 (6034), 11961201 .

28. Qian, L.; Winfree, E.; Bruck, J., Neural Network Computation with DNA Strand Displacement Cascades. Nature 2011, 475 (7356), 368.

29. Song, T.; Shah, S.; Bui, H.; Garg, S.; Eshra, A.; Fu, D.; Yang, M.; Mokhtar, R.; Reif, J., Programming DNA-Based Biomolecular Reaction Networks on Cancer Cell Membranes. J. Am. Chem. Soc. 2019.

30. Heinen, L.; Walther, A., Programmable Dynamic Steady States in Atp-driven Nonequilibrium DNA Systems. Sci. Adv. 2019, 5 (7), eaaw0590. 


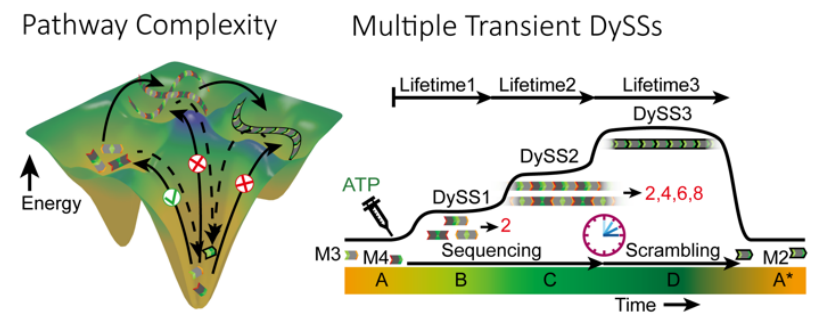

Table of Contents artwork 


\title{
Pathway Complexity in Fuel-Driven DNA Nanostructures with Autonomous Reconfiguration of Multiple Dynamic Steady States
}

\author{
Jie Deng ${ }^{\mathrm{a}-\mathrm{d}}$, and Andreas Walther ${ }^{\mathrm{a}-\mathrm{d} *}$ \\ ${ }^{a} A^{3}$ BMS Lab, Institute for Macromolecular Chemistry, University of Freiburg, Stefan-Meier-Straße 31, 79104 \\ Freiburg, Germany \\ b DFG Cluster of Excellence "Living, Adaptive and Energy-Autonomous Materials Systems" (livMatS), 79110 \\ Freiburg, Germany \\ ${ }^{c}$ Freiburg Materials Research Center, University of Freiburg, Stefan-Meier-Straße 21, 79104 Freiburg, Germany \\ ${ }^{d}$ Freiburg Center for Interactive Materials and Bioinspired Technologies, University of Freiburg, Georges-Köhler- \\ Allee 105, 79110 Freiburg, Germany
}

andreas.walther@makro.uni-freiburg.de 


\section{Materials and methods}

HPLC purified oligonucleotides were supplied by Integrated DNA Technologies Inc.. T4 DNA Ligase (HC, 20 Weiss Units $(\mathrm{WU}) / \mu \mathrm{L}$, recombinant $E$. coli strain) and BamHI (HC, 80 units $/ \mu \mathrm{L}$, Bacillus amyloliquefaciens $\mathrm{H}$ ) were supplied by Promega. ATP solution (10 mM in $1 \mathrm{mM}$ Tris-HCl pH 7.5) was purchased from Invitrogen. Agarose low EEO was supplied by PanReac AppliChem. Gene Ruler $1 \mathrm{k}$ bp and 50 bp DNA ladders (ready to use) were ordered from Thermo Scientific. Ethylenediaminetetraacetic acid disodium salt dihydrate (EDTA, biology grade) was supplied by CALBIOCHEM. Sodium chloride $(\mathrm{NaCl}, 99 \%)$ was ordered from ABCR. Tris (hydroxymethyl)aminomethane hydrochloride $\mathrm{pH} 8.0($ Tris- $\mathrm{HCl}$ ) and trizma base were ordered from Sigma-Aldrich. Acetic acid glacial (ACS, Reag. Ph. Eur. Analytical reagents) was supplied by VWR Chemicals. RotiR-GelStain (1,1'3,3',5,5'6,6'-Octamethyl-2,2'-spiro(2,3-dihydro-1H-Benzimidazol) was supplied by Carl Roth.

\section{Buffer compositions:}

T4 DNA Ligase Storage Buffer (Promega): $10 \mathrm{mM}$ Tris- $\mathrm{HCl}\left(\mathrm{pH} 7.4\right.$ at $\left.25^{\circ} \mathrm{C}\right), 50 \mathrm{mM} \mathrm{KCl}, 1 \mathrm{mM}$ Dithiothreitol (DTT), 0.1 mM EDTA, 50\% glycerol.

BamHI Storage Buffer (Promega): $10 \mathrm{mM}$ Tris- $\mathrm{HCl}$ (pH 7.4), $300 \mathrm{mM} \mathrm{KCl,} 5 \mathrm{mM} \mathrm{MgCl}$, $0.1 \mathrm{mM}$ EDTA, 1 mM DTT, $0.5 \mathrm{mg} / \mathrm{mL}$ BSA, $50 \%$ glycerol.

Restriction Enzyme 10x Buffer E (Promega): $60 \mathrm{mM}$ Tris-HCl (pH 7.5), $1 \mathrm{M} \mathrm{NaCl}, 60 \mathrm{mM} \mathrm{MgCl}, 10 \mathrm{mM}$ DTT. Annealing Buffer: $10 \mathrm{mM}$ TRIS- $\mathrm{HCl}(\mathrm{pH} 8.0), 50 \mathrm{mM} \mathrm{NaCl}$.

TAE Buffer: $40 \mathrm{mM}$ TRIS, $20 \mathrm{mM}$ acetic ccid, $1 \mathrm{mM}$ EDTA.

Quenching Buffer: 200 mM EDTA, 10 mM TRIS-HCl (pH 8.0), $50 \mathrm{mM} \mathrm{NaCl}$.

Supplementary Table 1: Oligonucleotide sequences. Sequences in red indicate the sticky ends for ligation.

\begin{tabular}{|c|c|c|c|c|}
\hline & ID & Oligonucleotide sequence & \#nt & Purification \\
\hline \multirow{2}{*}{ M1 } & $D_{1}$ & 5'-/5Phos/CCCTTACGGTGAATGGAATTCTCATGCGAATAGAGGAT-3' & 38 & HPLC \\
\hline & $D_{2}$ & 5'-/5Phos/GATCCTCTATTCGCATGAGAATTCCATTCACCGTAAGG-3' & 38 & HPLC \\
\hline \multirow{2}{*}{ M2 } & $D_{3}$ & 5'-/5Phos/GATCCCTTACGGTGAATGGAATTCTCATGCGAATAGAG-3' & 38 & HPLC \\
\hline & $D_{2}$ & 5'-/5Phos/GATCCTCTATTCGCATGAGAATTCCATTCACCGTAAGG-3' & 38 & HPLC \\
\hline \multirow{2}{*}{$\mathrm{C} 1$} & $\mathrm{D}_{4}$ & 5'-/5Phos/TCCCTTACGGTGAATGGAATTCTCATGCGAATAGAGGA-3' & 38 & HPLC \\
\hline & $D_{2}$ & 5'-/5Phos/GATCCTCTATTCGCATGAGAATTCCATTCACCGTAAGG-3' & 38 & HPLC \\
\hline \multirow{2}{*}{$\mathrm{C} 2$} & $D_{5}$ & 5'-/5Phos/ATCCCTTACGGTGAATGGAATTCTCATGCGAATAGAGG-3' & 38 & HPLC \\
\hline & $D_{2}$ & 5'-/5Phos/GATCCTCTATTCGCATGAGAATTCCATTCACCGTAAGG-3' & 38 & HPLC \\
\hline \multirow{2}{*}{ M3 } & $D_{6}$ & 5'-/5Phos/CCCTTACGGTGAATGGAATTCTCATGCGAATAGAG-3' & 35 & HPLC \\
\hline & $D_{2}$ & 5'-/5Phos/GATCCTCTATTCGCATGAGAATTCCATTCACCGTAAGG-3' & 38 & HPLC \\
\hline \multirow[t]{2}{*}{ M4 } & $D_{7}$ & $\begin{array}{l}\text { 5'-/5Phos/GATCCCTTACGGTGAATGGAATTCTCATGCGAATAGAGGAT- } \\
3^{\prime}\end{array}$ & 41 & HPLC \\
\hline & $D_{2}$ & 5'-/5Phos/GATCCTCTATTCGCATGAGAATTCCATTCACCGTAAGG-3' & 38 & HPLC \\
\hline
\end{tabular}




\section{Oligonucleotides:}

All DNA strands were HPLC purified and were used as received, to which certain amounts of annealing buffer were added to make stock solutions and the concentrations were calculated via UV-VIS spectroscopy. The doublestranded (ds) DNA tiles in this study were annealed from two complementary single-stranded (ss) DNA with the same stoichiometry at room temperature overnight. The annealed stock solutions were stored at $-20{ }^{\circ} \mathrm{C}$ for further use. In more details, $M 1$ was annealed from $D_{1}$ and $D_{2}$. M2 was annealed from $D_{3}$ and $D_{2}$. $C 1$ was annealed from $D_{4}$ and $D_{2}$. C2 was annealed from $D_{5}$ and $D_{2}$. M3 was annealed from $D_{6}$ and $D_{2}$. M4 was annealed from $D_{7}$ and $D_{2}$.

\section{Definition of Activity Units of both Enzymes}

Definition of the Weiss Unit to describe the activity of T4 DNA ligase (Promega): 0.01 Weiss Unit [WU] of T4 DNA Ligase is the amount of enzyme required to catalyze the ligation of greater than $95 \%$ of $1 \mu \mathrm{g}$ of $\lambda /$ HindIII fragments at $16^{\circ} \mathrm{C}$ in 20 minutes.

Unit definition to describe the activity of BamHI (Promega): One Unit [U] is defined as the amount of enzyme required to completely digest $1 \mu \mathrm{g}$ of lambda DNA in one hour at $37^{\circ} \mathrm{C}$ in $50 \mu \mathrm{L}$ assay buffer containing acetylated BSA added to a final concentration of $0.1 \mathrm{~g} / \mathrm{L}$.

\section{Transient polymerization of $\mathrm{M} 1$ and $\mathrm{M} 2$ for lag time investigation}

The experiment was performed at $25^{\circ} \mathrm{C}$ in $1 \times$ Promega Buffer E with $0.05 \mathrm{mM} \mathrm{M} 1$ (or M2), $0.46 \mathrm{WU} / \mu \mathrm{L}$ T4 DNA ligase, $0.1 \mathrm{mg} / \mathrm{mL}$ bovine serum albumin (BSA), 10 units $/ \mu \mathrm{L}$ BamHI, and $0.6 \mathrm{mM}$ ATP. At different time intervals, $6 \mu \mathrm{L}$ aliquots of the reaction solution were collected and quenched by $8 \mu \mathrm{L}$ of quenching buffer. The aliquots were then analyzed by $2 \mathrm{wt} . \%$ agarose gel electrophoresis (AGE) at $90 \mathrm{~V}$ for $2 \mathrm{~h}$ run time. The results were recorded by a transilluminator (Analytik Jena, Germany).

\section{Control experiments of transient polymerization of tiles with $\mathbf{2} \mathbf{n t}$ and $\mathbf{3} \mathbf{n t}$ overhangs}

The experiments were performed the same as above except $0.05 \mathrm{mM} \mathrm{C1}$ (or C2) was used for the transient polymerization. $6 \mu \mathrm{L}$ aliquots of the reaction solution were collected were collected and quenched by $8 \mu \mathrm{L}$ of quenching buffer after different time intervals for the first $5 \mathrm{~h}$ to check the kinetics of the transient polymerizations. Afterwards, the collected samples were analyzed by AGE (see above). Results in Figure S3.

\section{Pathway-controlled transient polymerization for transient multi-states}

The experiments were performed at $25^{\circ} \mathrm{C}$ in $1 \times$ Promega Buffer E with $0.025 \mathrm{mM} \mathrm{M} 3,0.025 \mathrm{mM} \mathrm{M} 4,0.46 \mathrm{WU} / \mu \mathrm{L}$ T4 DNA ligase, $0.1 \mathrm{mg} / \mathrm{mL}$ bovine serum albumin (BSA), 10 units $/ \mu \mathrm{L} \mathrm{BamHI}$, and 0.3, 0.6, or $0.9 \mathrm{mM}$ ATP. At different time intervals, $6 \mu \mathrm{L}$ aliquots of the reaction solution were collected and quenched by $8 \mu \mathrm{L}$ of quenching buffer. The collected samples were analyzed by AGE (see above).

\section{Disruption of dynamic steady-states for programmable transient multi-states}

The experiments were performed at $25^{\circ} \mathrm{C}$ in $1 \times$ Promega Buffer $\mathrm{E}$ with $0.05 \mathrm{mM}$ dsDNA tiles (M3, M4, and M2 in different ratios; 1:1:0, 5:5:2, and 1:1:1), $0.46 \mathrm{WU} / \mu \mathrm{L}$ T4 DNA ligase, $0.1 \mathrm{mg} / \mathrm{mL}$ bovine serum albumin (BSA), 10 units $/ \mu \mathrm{L} \mathrm{BamHI}$, and $0.6 \mathrm{mM}$ ATP. At different time intervals, $6 \mu \mathrm{L}$ aliquots of the reaction solution were collected and quenched by $8 \mu \mathrm{L}$ of quenching buffer. The collected samples were analyzed by AGE (see above). 


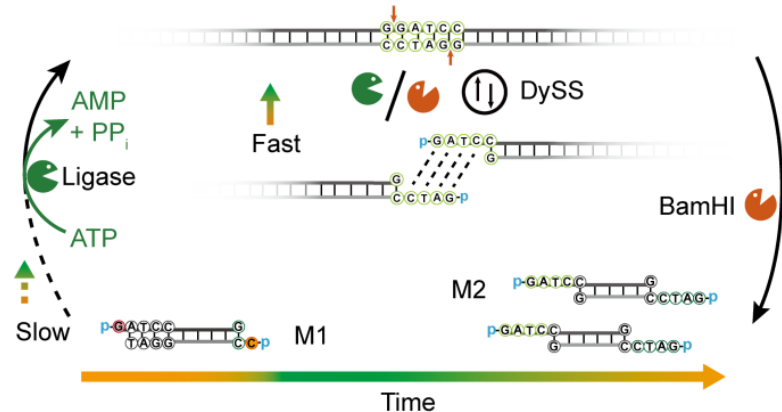

b

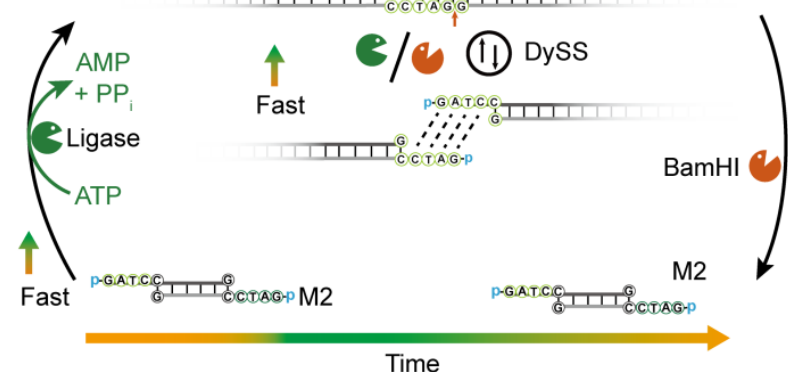

Figure S1. Evolution of sticky ends during the fuel-driven operation of the ERN. (a) Schematic illustration of transient polymerization of $1 \mathrm{nt}$ sticky-end dsDNA tiles. Reconfiguration of the $1 \mathrm{nt}$ ends into $4 \mathrm{nt}$ ends occurs. (b) Schematic illustration of transient polymerization of 4 nt sticky-end dsDNA tiles. No reconfiguration of the sticky ends occurs.
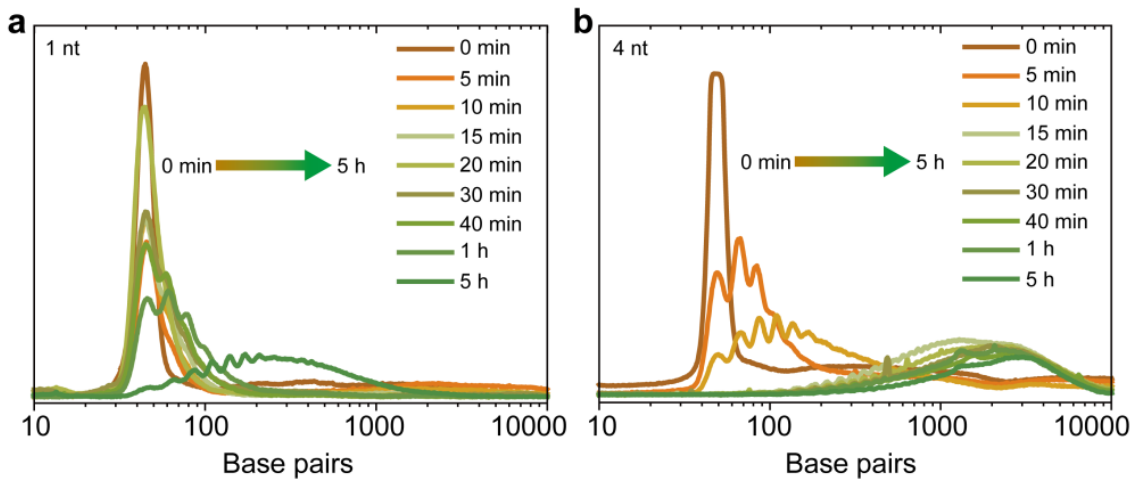

Figure S2. Length of the sticky ends controls the lag time in ATP-driven, dynamic covalent DNA assembly. (a-b) Gray scale profiles from AGE for the first $5 \mathrm{~h}$ of transient DySS polymerization of (a) M1 and (b) M2. The results further show the kinetics of the band shifts for both polymerizations. The polymerization of $\mathrm{M} 1$ is not obvious at the first hour, and the elongation of the DNA polymer is very slow. Only after $5 \mathrm{~h}$, there is obvious gel band shift. However, the chains for the M2 system rapidly grow to ca. 800 bp within 10 min and reach a DySS plateau at ca. $9000 \mathrm{bp}$ after $15 \mathrm{~min}$.
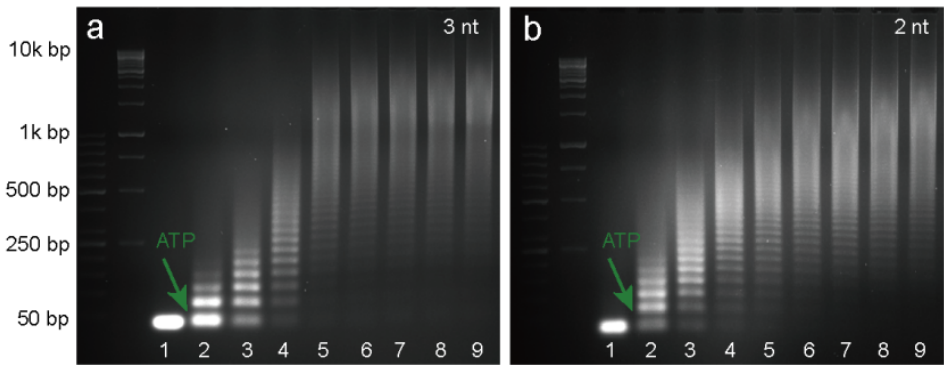

Figure S3. Lower lag times and less pronounced lag phases for $\mathbf{2} \mathrm{nt}$ (C1) and $\mathbf{3} \mathrm{nt}$ (C3) sticky ends on dsDNA monomers. AGE analysis for the transient DySS polymerization of (a) $3 \mathrm{nt}$ and (b) 2 nt-overhang dsDNA tiles. Standard dsDNA tiles in the enzymatic reaction network have an overhang of 4 nucleotide (nt) for ligation ( $5^{\prime}$-GATC/GATC- $3^{\prime}$ ), which has 10 pairs of hydrogen bonds. When the overhang is shortened to $3 \mathrm{nt}\left(5^{\prime}-\mathrm{ATC} / \mathrm{GAT}-3^{\prime}\right)$ and $2 \mathrm{nt}\left(5^{\prime}-\mathrm{TC} / \mathrm{GA}-3^{\prime}\right)$, that have 7 and 5 pairs of hydrogen bonds, respectively, there is no obvious lag time as compared to the building blocks having an overhang of $1 \mathrm{nt}\left(5^{\prime}-\mathrm{C} / \mathrm{G}-3^{\prime}\right)$ with 3 pairs of hydrogen bonds. (Figure 2 in the main MS). Thus $1 \mathrm{nt}$ overhangs were used throughout this study for significant lag time and pathway control. Conditions: $25^{\circ} \mathrm{C}, 1 \times$ Promega Buffer E, $0.05 \mathrm{mM}$ dsDNA (C1 or C2), $0.46 \mathrm{WU} / \mu \mathrm{L}$ T4 DNA ligase, 10 units/ $\mu \mathrm{L}$ BamHI, and 0.6 mM ATP. Lane assignment: 1: $0 \mathrm{~min}, 2: 5 \mathrm{~min}, 3: 10$ $\min , 4: 15 \min , 5: 20 \min , 6: 30 \mathrm{~min}, 7: 40 \mathrm{~min}, 8: 1 \mathrm{~h}, 9: 5 \mathrm{~h}$. 